\title{
Dynamic experimental studies of A6N01S-T5 aluminum alloy material and structure for high-speed trains
}

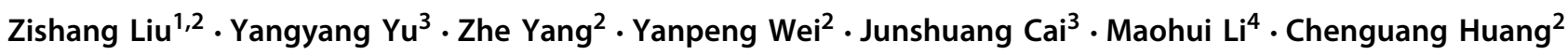

Received: 20 August 2018 / Revised: 19 October 2018 / Accepted: 14 November 2018 / Published online: 2 May 2019

(C) The Chinese Society of Theoretical and Applied Mechanics and Springer-Verlag GmbH Germany, part of Springer Nature 2019

\begin{abstract}
In this study, we focus on the dynamic failure property of A6N01S-T5 aluminum alloy use for high-speed trains. The method of split Hopkinson tensile bar (SHTB) and three-dimensional (3D) digital image correlation (DIC) was put forward to find the dynamic mechanical properties and dynamic failure strain of A6N01S-T5 aluminum alloy, and on the basis of this, Johnson-Cook model constitutive parameters and dynamic failure strain parameters were obtained through a series of static and dynamic tests. An important character of this method was that the sandwich structure from the true high-speed train was used in penetration test, followed by the numerical calculation of the same working condition using LS-DYNA. Then we compare the experimental results with simulation results mentioned above in terms of failure morphology in structure and the bullet speed throughout the entire process to verify the accuracy of the parameter. The experimental results provide a data basis for the crash simulation model of high-speed trains, in turn to optimize the structural design and whole efficiency.
\end{abstract}

Keywords A6N01S-T5 aluminum alloy · Dynamic failure strain · Constitutive model · Dynamic mechanical properties

\section{Introduction}

The 6xxx aluminum alloy has been widely used in the production of high-speed trains [1-5]. Aluminum alloy's property can be changed by adding other elements [6]. The aluminum alloys, which mainly add magnesium and silicon, are called 6xxx aluminum alloys [7]. The main addition elements of 6xxx aluminum alloy form a $\mathrm{Mg}_{2} \mathrm{Si}$ phase [8]. The 6xxx aluminum alloy has high strength, plasticity, and elongation, as well as good formability, weldability, and corrosion resistance [9-14].

Creating a lighter vehicle body is an important trend in the design of high-speed trains [15-17]. Reducing the weight of the car body can reduce the consumption of resources and improve the speed of the train $[18,19]$. The $6 \mathrm{xxx}$ alu-

Yanpeng Wei

weiyanpeng@imech.ac.cn

1 Institute of Mechanics, Chinese Academy of Sciences, Beijing 100190, China

2 University of Chinese Academy of Sciences, Beijing 100049, China

3 CRRC Qingdao Sifang CO., LTD, Qingdao 266111, China

4 Quartermaster Research Institute of Engineering and Technology, Beijing 100010, China minum alloy is an ideal energy absorbing material. When the structure is impacted, the energy absorption of the 6xxx aluminum alloy (about $55 \mathrm{~kJ} / \mathrm{kg}$ ) is larger than that of conventional steel $(30 \mathrm{~kJ} / \mathrm{kg})$ [20-22]. The $6 \mathrm{xxx}$ aluminum alloy can be strengthened by heat treatment [23]. The selected processing technology will eventually affect the mechanical properties of the aluminum alloy [24-26]. T5 is a common heat treatment process for the $6 \mathrm{~N} 01$ aluminum alloy used in high-speed train bodies [1, 2].

Regarding the mechanical properties of the 6xxx aluminum alloy, much research has been done by scholars in the past. For example, Shi et al. [27] carried out compression experiments on the 6xxx aluminum alloy. In the scope of the study, compression constitutive models are proposed. Also, Zhu et al. [28] performed medium strain rate tensile tests on the 6xxx aluminum alloy. The stress-strain curves and failure modes of materials were studied. The work of Vilamosa et al. [29] presented tensile tests in a wider range of strain rate $\left(0.01-750 \mathrm{~s}^{-1}\right)$ and temperature range $\left(20-350{ }^{\circ} \mathrm{C}\right)$ to study the joint effect of two factors. The results show that the material exhibits negligible strain rate sensitivity at temperatures below $200{ }^{\circ} \mathrm{C}$. The strain rate sensitivity at higher temperatures should not be ignored. To this end, Zhang et al. [30] used a split Hopkinson tensile bar (SHTB) to conduct quasistatic and dynamic tensile tests on the 7xxx aluminum alloy. 
The stress-strain curves of materials at different strain rates were obtained. The authors have made appropriate improvements based on the Johnson-Cook constitutive theory. The parameters involved in the constitutive model are fitted by experimental results. The failure strain values under different strain rates are brought into the damage and fracture criterion formula to obtain the dynamic failure strain. The specific dynamic failure parameters are fitted. The numerical simulation analysis of tension test of the specimen is carried out by using the fitted constitutive model.

The failure strain of the aluminum alloy is affected by many factors [31-33]. The Johnson-Cook model involves a total of five damage parameters D1-D5. The five parameters concern the effects of stress state, strain rate and temperature. Only if more experiments under different stress triaxialities and temperature are carried out can all the five parameters be obtained [34].

In the past, the main way to get dynamic failure strain is to fit the engineering failure strain under different working conditions. It is mainly indirect rather than direct. The validity of the parameters is verified by the tensile test. The verification method is not accurate enough compared to the test of real impact conditions at the structural level.

In view of the shortcomings of the previous research on dynamic failure strain acquisition and parameter verification, we put forward the SHTB + three-dimensional (3D) digital image correlation (DIC) method to obtain dynamic mechanical properties and dynamic failure strain the of A6N01S-T5 aluminum alloy. Through a series of static and dynamic tests for the A6N01S-T5 aluminum alloy, we obtained the Johnson-Cook constitutive model parameters and dynamic failure strain parameters. We have conducted penetration tests on sandwich structures used on real highspeed trains. The experimental results are compared with the simulation results. The accuracy of the parameters is verified by comparing the failure morphology of the structure and the velocity-time curve of the bullet. The experimental results provide a data basis for the crash simulation model of high-speed trains. The efficiency of structural design and optimization is improved.

\section{Experimental method}

\subsection{Static mechanical performance analysis}

First of all, the static test of the material is carried out. The specimen was cut from the A6N01S-T5 aluminum alloy plate material supplied by CRRC Qingdao Sifang Co., Ltd. As shown in Fig. 1, it was processed into a static tensile test piece after wire cutting. The surface of the specimen is sanded with 1000 grit sandpaper, and the angle of the specimen is smoothed by grinding process.

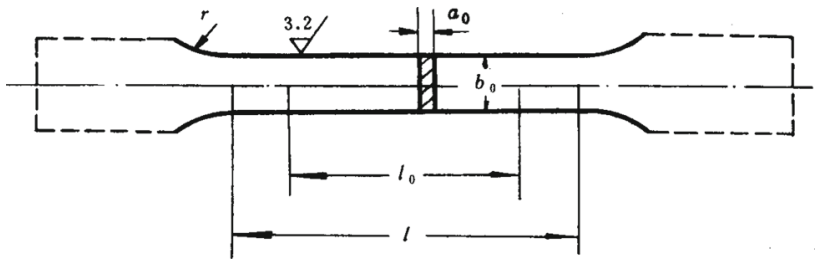

Fig. 1 Static tensile specimen of the A6N01S-T5 aluminum alloy

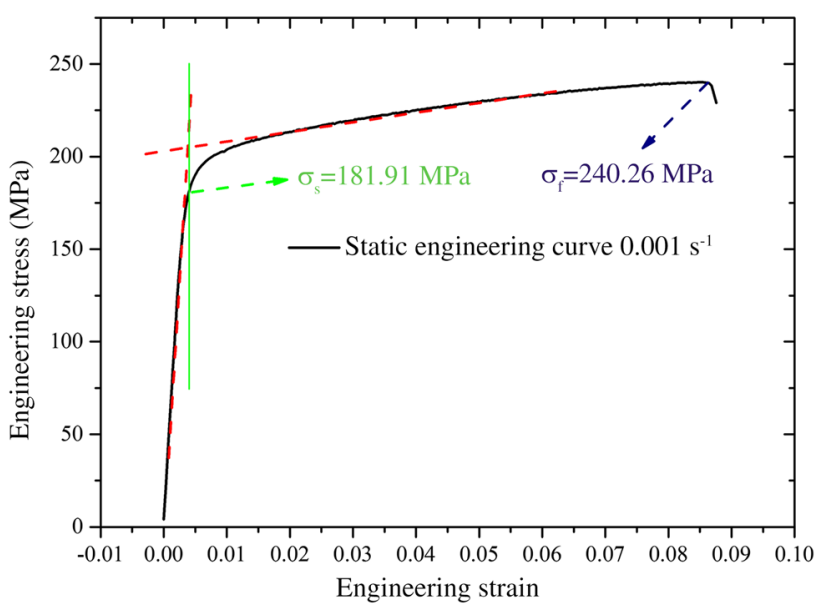

Fig. 2 Static stress-strain curve of the A6N01S-T5 aluminum alloy

Here, $a_{0}=2 \mathrm{~mm}, b_{0}=15 \mathrm{~mm}, r=25 \mathrm{~mm}, l_{0}=30 \mathrm{~mm}$, and $l=45 \mathrm{~mm}$. The surface roughness is 3.2 .

Static tension is applied with a strain rate of $0.001 \mathrm{~s}^{-1}$. The typical tensile curve is shown in the Fig. 2.

From Fig. 2, we can see that the A6N01S-T5 aluminum alloy is a typical elastic-plastic material with good ductility, and the plastic flow stress has an increasing trend with the increase of strain. The experiment adopts the slope method to determine the yield strength of the engineering because there is no upper yield point and lower yield point in the curve. The method is to extend the slope of the typical elastic segment upwards, while extending the slope of the plastic flow segment to the left. The intersection of the two extension lines is defined as the strain at the time of yield. From Fig. 2, it can be accurately determined that the static engineering yield strength of the A6N01S-T5 aluminum alloy is $181.91 \mathrm{MPa}$. For the engineering fracture strength of the material, the highest point in the curve is used for the determination. And the final engineering fracture strength is determined to be 240.26 MPa in Fig. 2. In the static curve, no softening segment was observed, but the fracture occurred immediately after reaching the maximum engineering fracture strength. Therefore, the fracture strain value of the material is $8.6 \%$, which corresponds to the strain of the maximum engineering fracture strength. It is worth noting that the fracture strain is not the actual strain of the material, but the average effect of the engineering result. Because of the necking effect and 


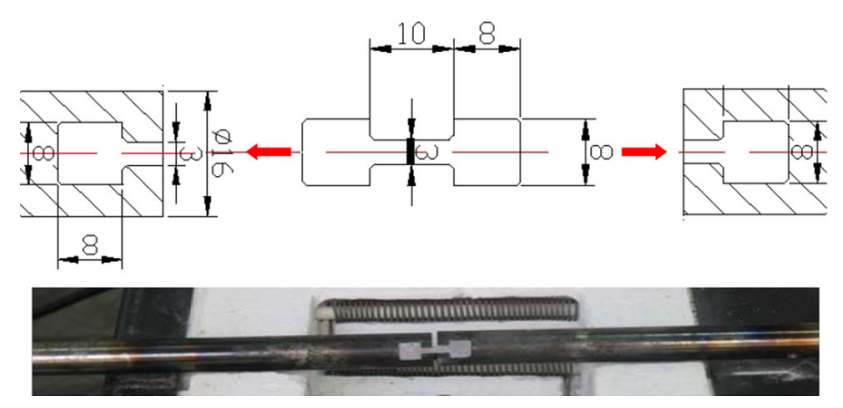

Fig. 3 Flat specimen and its connection of the A6N01S-T5 aluminum alloy

non-uniform deformation in the large deformation stage, the failure strain of the actual material is larger than that of the engineering fracture. The real dynamic failure strain is the focus of this experiment, which will be explained in the following chapters.

\subsection{Analysis of dynamic mechanical properties}

In the SHTB test technology, the contact and connection of the test piece is a key technology. Especially for the tensile bar device, the stress wave is transmitted through the connection part. The connection method directly affects the accuracy of the test and the validity of data. Dynamic tensile test specimens generally have cylindrical and flat shapes. For cylindrical specimens, most use threaded connection. Although this method will also have a certain impact on the transmission of stress waves, it has been proved that in the incident wave with larger magnitudes, the effect is relatively small and acceptable. There are generally two types of flat connection methods. One is to use a high-strength adhesive to attach a flat test piece to a slot of the bar. Although this method can obtain a good stress waveform, the glue process is complicated and the test cycle is long. It takes approximately $2-3 \mathrm{~h}$ to prepare the test piece for the first test; the other connection method is to use a pin to connect. Although the test efficiency is high, the strength of the bar is greatly reduced and the stress waveform is greatly influenced by the stress concentration area in the head of the bar.

Because of the limitation of the shape of the welded part, the specimen can only be processed into a flat shape. In order to obtain better test data, this paper abandons the connection method of the flat test specimen in the past. We adopts the self-designed card sleeve connection method. The sample's thickness is $2 \mathrm{~mm}$, as shown in Fig. 3.

In order to determine the strain rate effect on the materials, the dynamic tensile tests were conducted in the low strain rate range $\left(300-600 \mathrm{~s}^{-1}\right)$, medium strain rate range (600-1000 $\mathrm{s}^{-1}$ ), and high strain rate range (greater than $\left.1000 \mathrm{~s}^{-1}\right)$.

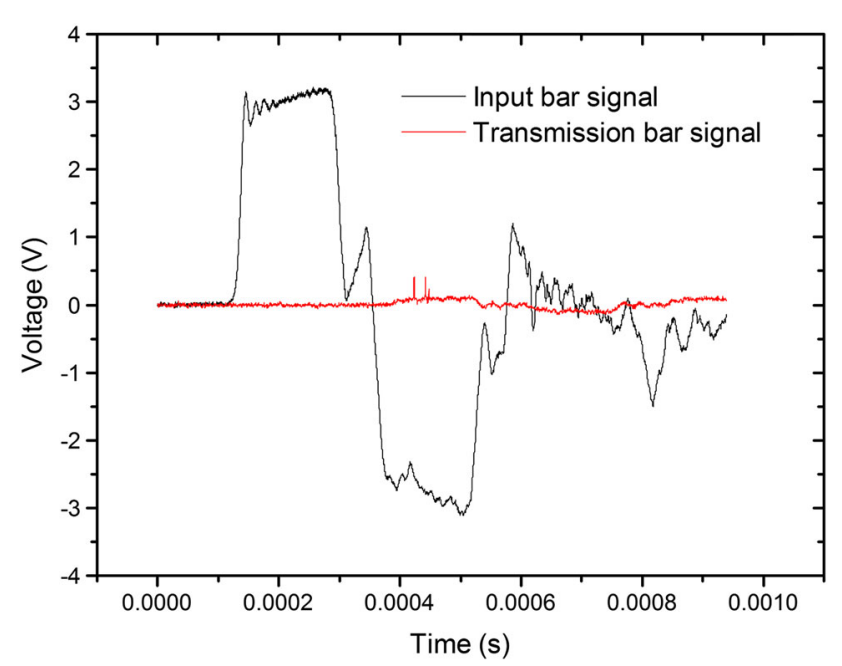

Fig. 4 Original voltage-time signal at $1200 \mathrm{~s}^{-1}$

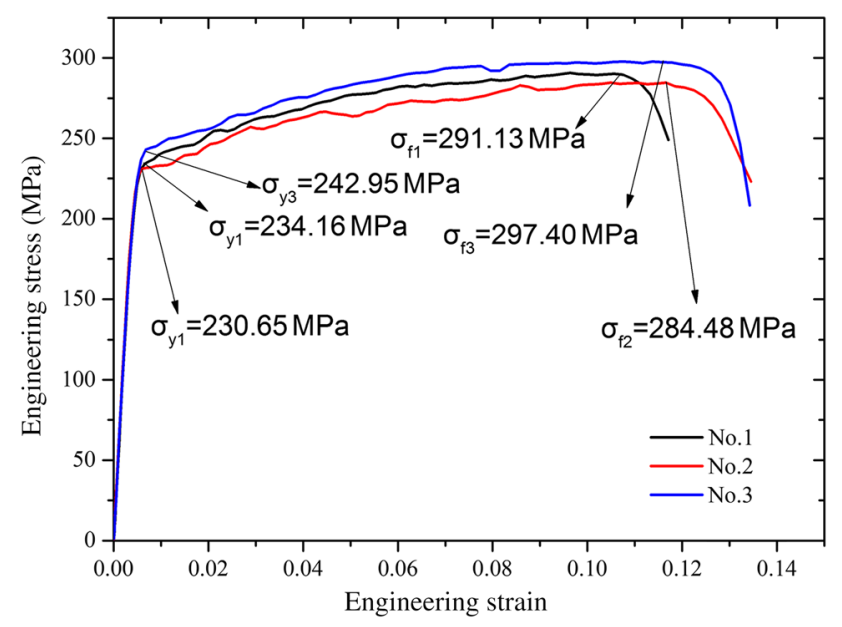

Fig. 5 Repeated tests with variable rate at $1200 \mathrm{~s}^{-1}$

The signals of input bar and transmission bar obtained at the strain rate of $1200 \mathrm{~s}^{-1}$ are shown in Fig. 4.

As show in Fig. 5, the repeatability analysis was carried out under the condition of variable rate of about $1200 \mathrm{~s}^{-1}$. The repeatability of the experiment was demonstrated by $\sigma_{\mathrm{f}}$ and $\sigma_{\mathrm{y}}$. The difference between the maximum relative deviation $\sigma_{\mathrm{f}}$ and the average value is $2.2 \%$, while the difference of the $\sigma_{\mathrm{y}}$ is $3.0 \%$.

Figures 6, 7, and 8 show the dynamic tensile curves of the A6N01S-T5 aluminum alloy at different strain rates.

From Fig. 8, we can see that the A6N01S-T5 aluminum alloy not only has the typical strain-strengthening effect, but also has a relatively obvious strain rate strengthening effect. Because there is no upper yield point or lower yield point in the curve, we also adopt the slope method to determine the engineering yield strength. From Figs. 6, 7, and 8 , the dynamic engineering yield strengths of the A6N01ST5 aluminum alloy at $320 \mathrm{~s}^{-1}, 720 \mathrm{~s}^{-1}$, and $1200 \mathrm{~s}^{-1}$ can 


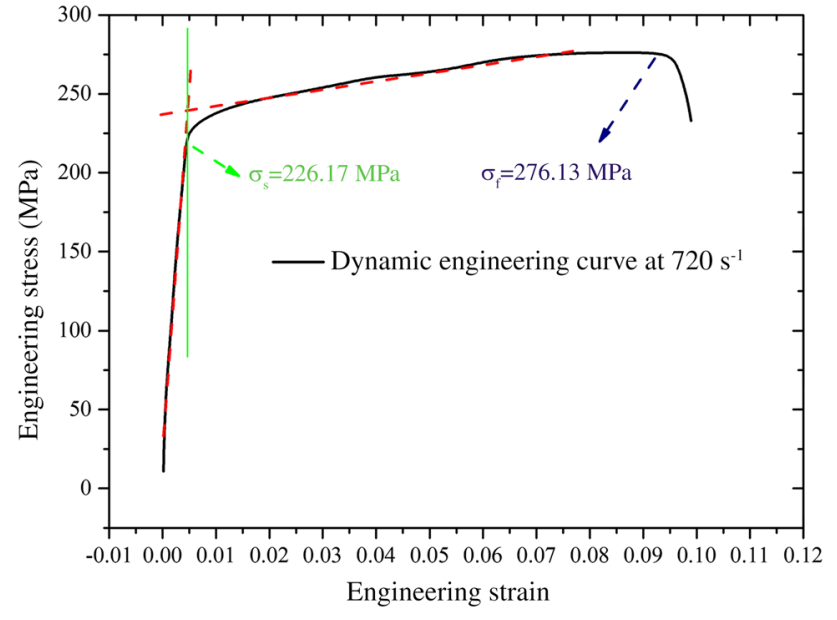

Fig. 6 Dynamic stress-strain curves of the A6N01S-T5 aluminum alloy at $320 \mathrm{~s}^{-1}$

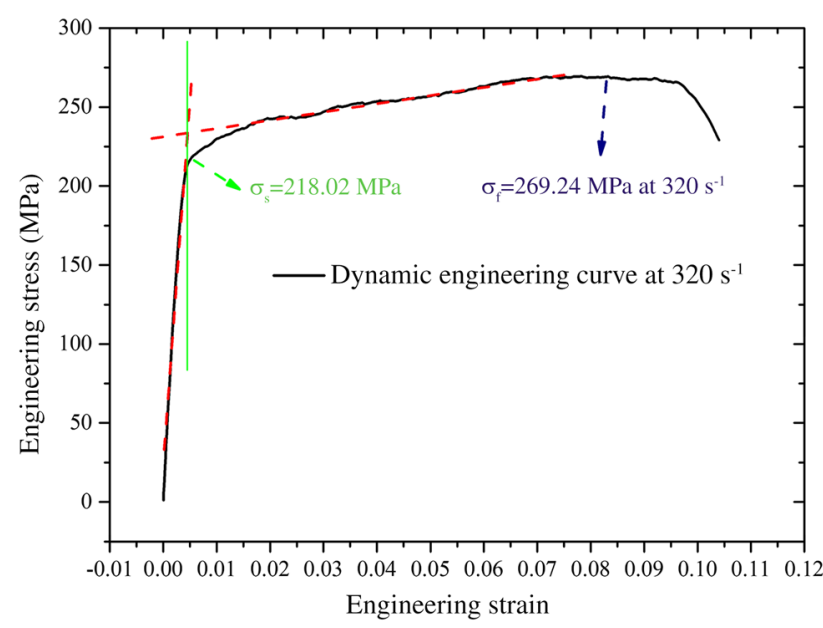

Fig. 7 Dynamic stress-strain curves of the A6N01S-T5 aluminum alloy at $720 \mathrm{~s}^{-1}$

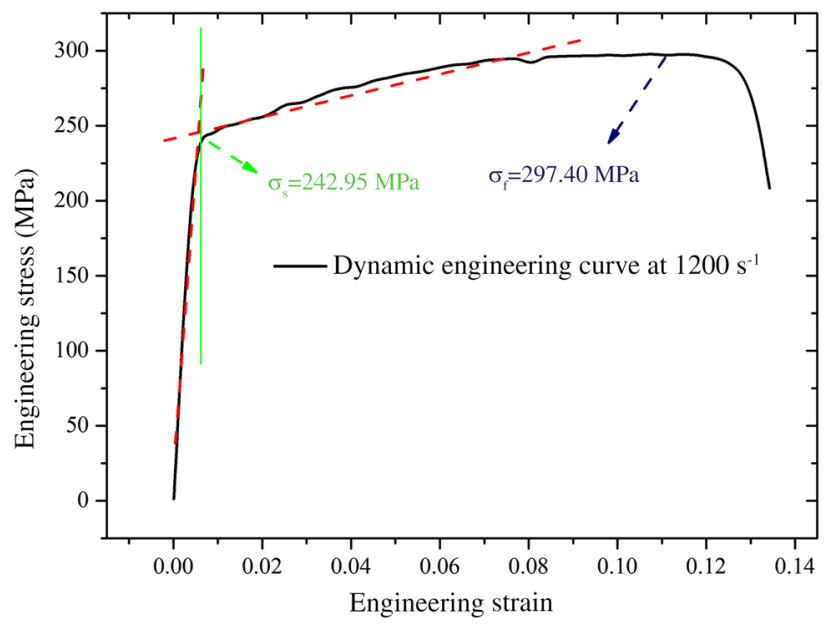

Fig. 8 Dynamic stress-strain curves of the A6N01S-T5 aluminum alloy at $1200 \mathrm{~s}^{-1}$ be accurately determined to be 218.02 $\mathrm{MPa}, 226.17 \mathrm{MPa}$, and $242.95 \mathrm{MPa}$, respectively. For the engineering fracture strength of the material, the highest point in the curve is used to determine. In Figs. 6, 7 and 8, it can be determined that the final engineering fracture strength is 269.24 MPa, $276.13 \mathrm{MPa}$, and 297.40 $\mathrm{MPa}$ at $320 \mathrm{~s}^{-1}, 720 \mathrm{~s}^{-1}$, and $1200 \mathrm{~s}^{-1}$.

\section{Dynamic constitutive parameter fitting}

We use the Johnson-Cook constitutive relationship for high-speed train body materials to perform the strain ratedependent constitutive description. Since the ambient temperature softening effect of materials is not involved in this project, the following simplified form is used

$\bar{\sigma}_{\mathrm{p}}=\left(A+B \bar{\varepsilon}_{\mathrm{p}}^{n}\right)\left(1+C \ln \frac{\dot{\varepsilon}_{\mathrm{p}}}{\dot{\varepsilon}^{*}}\right)$.

where $\bar{\sigma}_{\mathrm{p}}$ is the plastic equivalent flow stress, which is a scalar describing the plastic stress bias, defined as

$\bar{\sigma}_{\mathrm{p}}=\sqrt{\frac{1}{2}\left[\left(\sigma_{1}-\sigma_{2}\right)^{2}+\left(\sigma_{3}-\sigma_{2}\right)^{2}+\left(\sigma_{1}-\sigma_{3}\right)^{2}\right]}$,

$J_{2}$ is the second stress invariant, and $\sigma_{1}, \sigma_{2}$, and $\sigma_{3}$ are the first principal stress, the second principal stress, and the third principal stress, respectively. In the case of uniaxial stretching, $\sigma_{2}=\sigma_{3}=0$, so

$\bar{\sigma}_{\mathrm{p}}=\sigma_{1}=\sigma$

That is, the plastic equivalent stress is equal to the uniaxial tensile stress.

$\bar{\varepsilon}_{\mathrm{p}}$ is the plastic equivalent flow strain, which is a scalar describing the amount of plastic strain, defined as

$\bar{\varepsilon}_{\mathrm{p}}=\frac{\sqrt{2}}{3} \sqrt{\left[\left(\varepsilon_{1}-\varepsilon_{2}\right)^{2}+\left(\varepsilon_{3}-\varepsilon_{2}\right)^{2}+\left(\varepsilon_{1}-\varepsilon_{3}\right)^{2}\right]}$.

Among them, $J_{2}^{\prime}$ is the second strain partial invariant, $\varepsilon_{1}$, $\varepsilon_{2}$, and $\varepsilon_{3}$ are the first principal strain, the second principal strain, and the third principal strain, respectively. During uniaxial stretching

$\varepsilon_{1}=\varepsilon, \quad \varepsilon_{2}=\varepsilon_{3}=-\frac{1}{2} \varepsilon$.

Therefore, the final uniaxial tensile strain is axial strain.

$\dot{\varepsilon}^{*}$ is the reference strain rate. Its value is generally taken as 1 , and $\dot{\varepsilon}_{\mathrm{p}}$ is the plastic strain rate. In the model, $A, B$, and $n$ are static parameters, which are given by fitting the static stress-strain curve. $C$ is the strain rate parameter, which is obtained by fitting curves to different strain rates. 


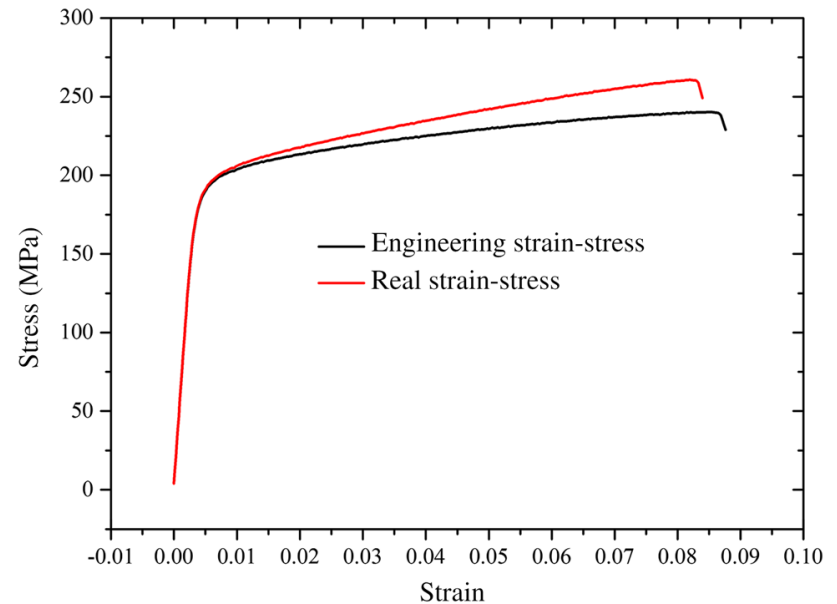

Fig. 9 True stress-strain curves and engineering stress-strain curves

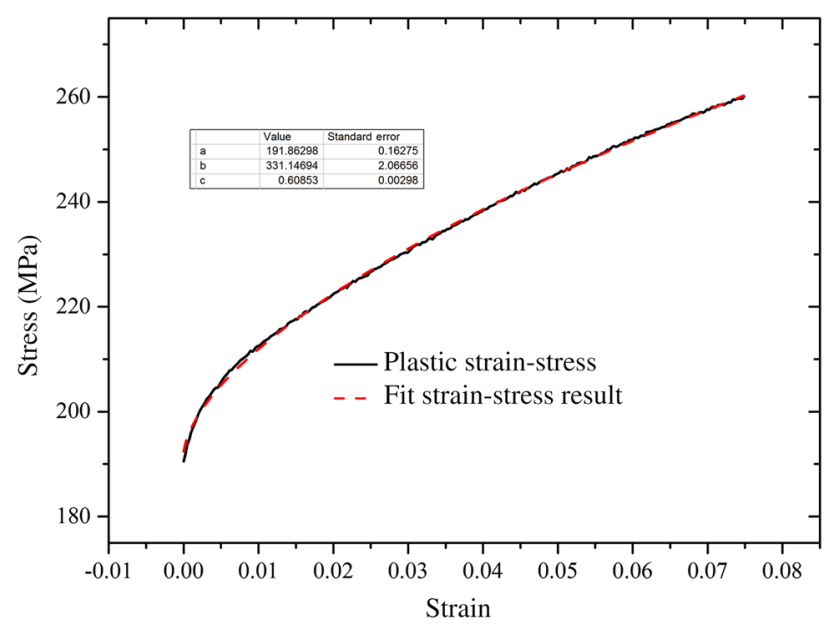

Fig. 10 Fitting of static parameters

\subsection{Fitting of static parameters}

To fit $A, B$, and $n$, the static stress-strain curve needs to be pre-processed. The first step is to transform the engineering stress-strain curve into a true stress-strain curve. Follow these two formulas

$\varepsilon_{\text {real }}=\ln \left(1+\varepsilon_{\text {eng }}\right)$,

$\sigma_{\text {real }}=\varepsilon_{\text {eng }}\left(1+\varepsilon_{\text {eng }}\right)$.

Figure 9 shows the conversion results of the static stress-strain curve. From Fig. 9, the plastic flow section of the material is selected for fitting. It is worth pointing out that the constitutive model $\bar{\varepsilon}_{\mathrm{p}}$ is the plastic flow strain, so the elastic strain should be subtracted in the graph first. Then we use exponential function $A+B x^{n}$ to fit and give the fitting result and error of three parameters, as shown in Fig. 10.

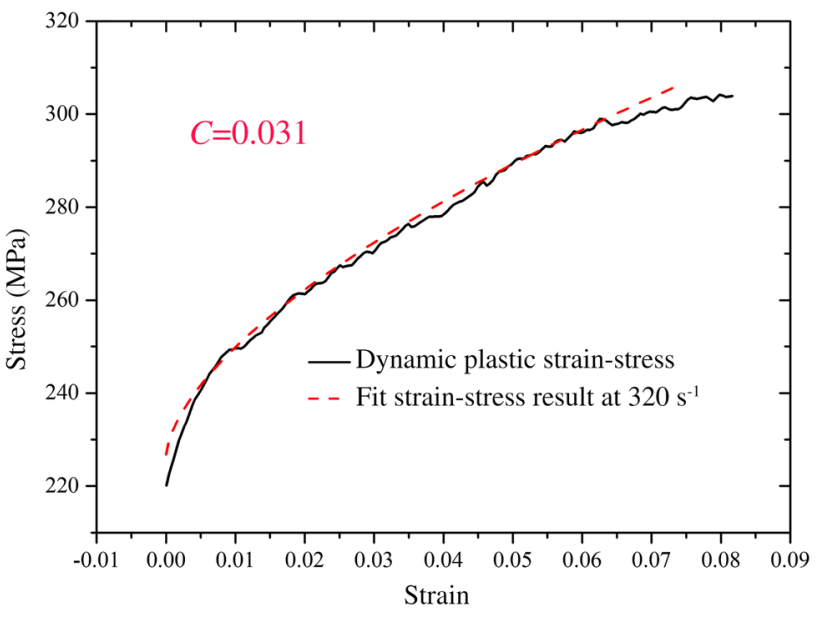

Fig. 11 Fitting of dynamic parameters

As Fig. 10 shows, we get the fitting result of three parameters, $A=191.862, B=331.146, n=0.609$.

\subsection{Dynamic parameter fitting}

This experiment uses a relatively simple and practical method for dynamic parameter fitting. A dynamic stress-strain curve is used to fit the parameters, and then this parameter is used to compare the other conditions. The dynamic stress-strain curve should also be transformed into a true stress-strain curve. The elastic strain section is subtracted to obtain the plastic flow stress-strain relationship. Then we use formula $y=d\left(A+B x^{n}\right)$ to fit, where $A, B$, and $n$ are selected as fixed parameters, and $d$ is a variable parameter. Finally, the value of $C$ can be obtained by the following formula

$C=(d-1) / \ln \left(\frac{\dot{\varepsilon}_{\mathrm{p}}}{\dot{\varepsilon}^{*}}\right)$.

In the selection of the fitting strain rate, we select the low strain rate conditions that are in line with the magnitude of the vehicle's crash strain rate, which can effectively ensure the validity of the model parameters in the future numerical calculation (Fig. 11).

From the fitting results, we can obtain $C=0.031$. The results are compared with other strain rate data. From the results, the series of parameters can effectively reflect the dynamic mechanical behavior of aluminum alloy (Fig. 12).

From this, we have obtained the Johnson-Cook constitutive parameters of the A6N01S-T5 aluminum alloy, as follows

$A=191.862, \quad B=331.146, n=0.609, \quad C=0.031$. 


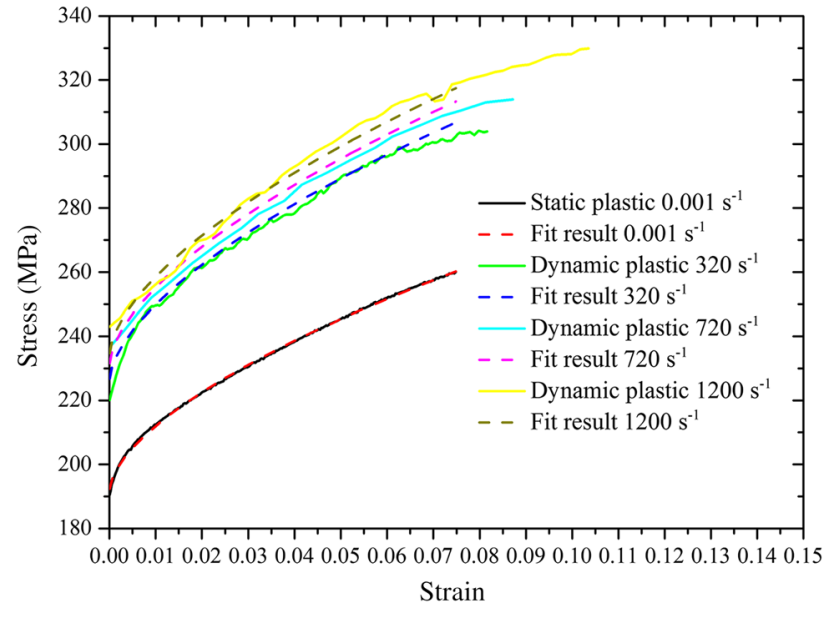

Fig. 12 Fitting results under different strain rates

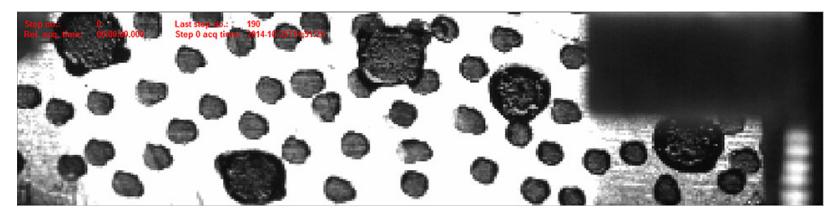

Fig. 13 Speckled specimen of the A6N01S-T5 aluminum alloy

\section{Acquisition of dynamic failure strain}

\subsection{Preparation of the dynamic DIC specimen}

The dynamic failure strain in this experiment is acquired by using high-speed DIC technology. The most important process is the preparation of speckled specimens. The conventional sprayed speckles will fall off during the large deformation stage. Overall, the spray speckle is not suitable for dynamic large deformation. The original speckle preparation process of this experiment not only ensures that it does not fall off under large deformation, but also its interface strength is extremely high. It will not fall off under the action of impact load. The facts show that the speckle preparation method used in this experiment can effectively capture the dynamic strain field history of materials before large deformation and failure. The prepared speckle specimens are shown in Fig. 13.

The high-speed image acquisition unit includes a highspeed charge coupled device (CCD) camera and a highbrightness light source. The trigger unit is responsible for the accurate triggering of high-speed camera image acquisition. The high-speed CCD camera is responsible for highfrequency shooting. Since the exposure time is very short, a strong light source is required to illuminate the surface of the test piece to ensure that the image has a certain brightness. The whole set of devices is shown in Fig. 14.

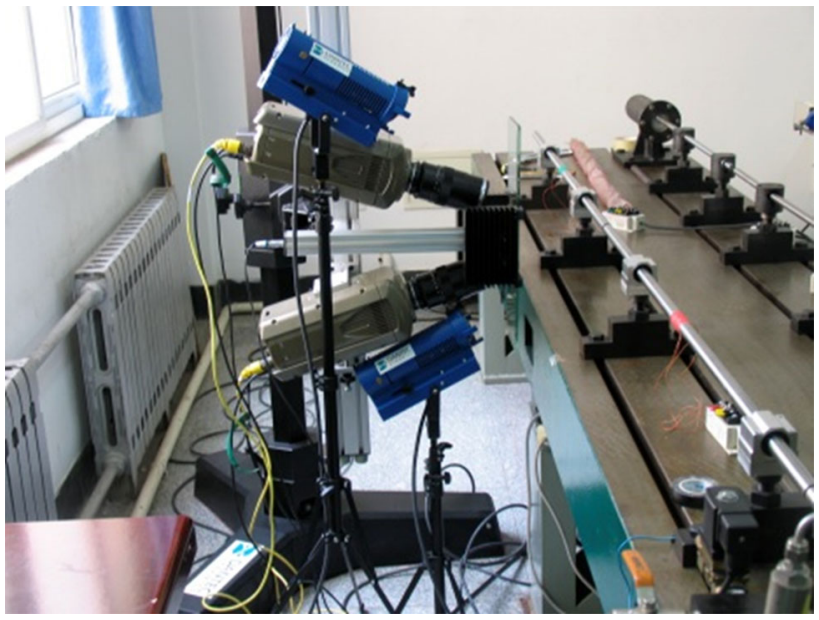

Fig. 14 High speed photography system

\subsection{Analysis of dynamic failure strain}

Figure 15 shows the dynamic stretching and final failure of the material, which was taken by high-speed photography. It can be seen that the material has contracted and localized large deformations at the last extreme of stretching. The traditional strain treatment has not been able to obtain local correct deformation information. As shown in the Fig. 15, the fracture occurred in the middle of the specimen, and the speckle morphology remained intact.

By using our own DIC processing program, we can obtain the distribution of the strain field during the entire dynamic deformation process. We select the deepest part of the cracking position as the research object in the strain cloud diagram. We can obtain the main strain history in the two directions at this point, which are shown in Fig. 16.

At the final fracture of the material, we can obtain that $\varepsilon_{1}$ $=0.17087, \varepsilon_{2}=-0.03387$ from the Fig. $16 . \varepsilon_{2}=\varepsilon_{3}$ is still satisfied in the neck segment. We know that stress and strain are all second-order tensors. Ignoring the part of the spherical tensor, we only consider partial tensor. We can introduce the concept of equivalent stress and equivalent strain. The equal effect changes are considered as failure strain here

$\varepsilon_{\mathrm{eff}}=\frac{\sqrt{2}}{3} \sqrt{\left(\varepsilon_{1}-\varepsilon_{2}\right)^{2}+\left(\varepsilon_{2}-\varepsilon_{3}\right)^{2}+\left(\varepsilon_{3}-\varepsilon_{1}\right)^{2}}$.

Substituting the value of the corresponding amount into this formula yields

$\varepsilon_{\text {eff }}=0.137=13.7 \%$. 


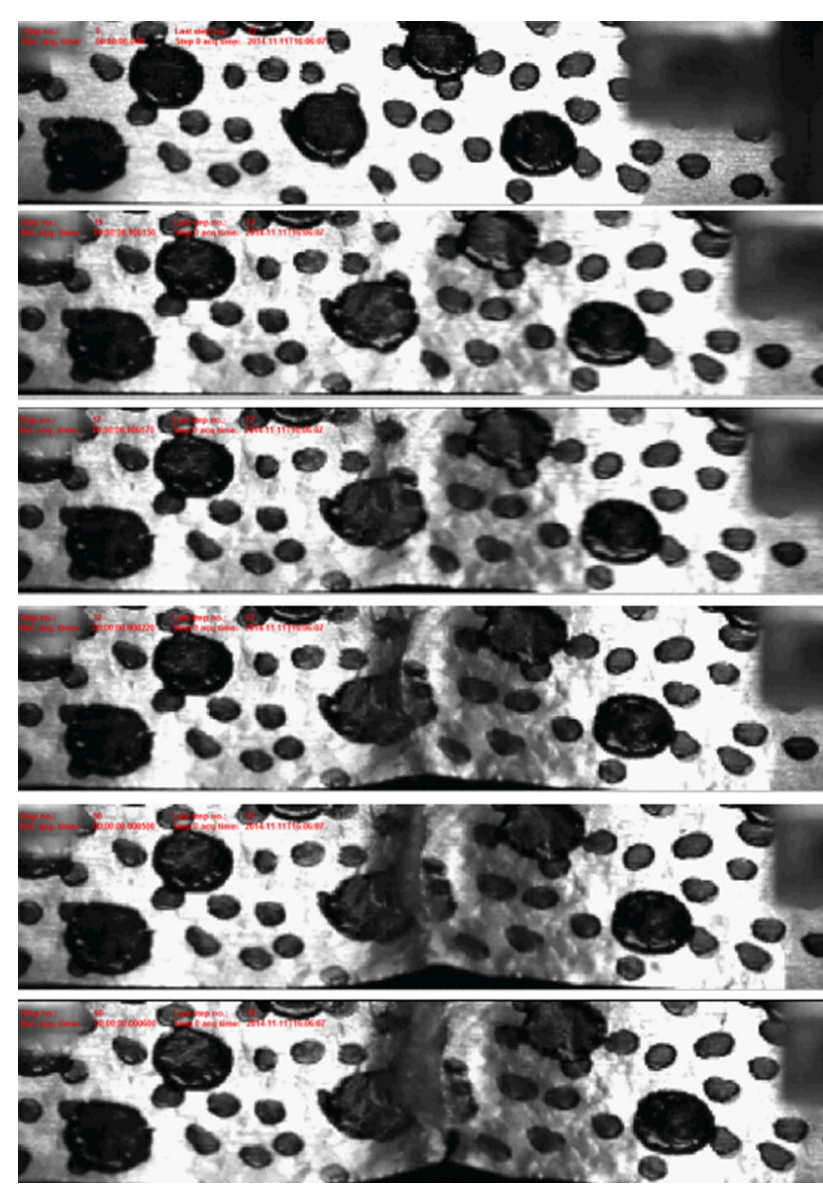

Fig. 15 Dynamic fracture process of the A6N01S-T5 aluminum alloy

\section{Verification of obtained parameters}

In the previous part, we obtained the Johnson-Cook constitutive model parameters of the A6N01S-T5 aluminum alloy. This chapter validates the validity of the dynamic constitutive parameters and dynamic failure strain parameters. Using the comparison of numerical results and experimental results, the accuracy of material constitutive parameters and failure parameters was determined.

The constitutive parameters of the A6N01S-T5 aluminum alloy were obtained by experiment and parameter fitting. We obtained that $A=191.862 \mathrm{MPa}, B=331.146 \mathrm{MPa}, n=0.609$, $C=0.031$ and dynamic failure strain $\varepsilon_{\text {eff }}=0.137$. We substituted these parameters into LS-DYNA software to analyze. The calculation model uses the same loading and boundary conditions as the real test case. After the calculation and experimental results are obtained, the two results are compared and analyzed. The accuracy of constitutive parameters and dynamic failure strain parameters of the A6N01S-T5 aluminum alloy are verified by comparison of morphology and velocity.
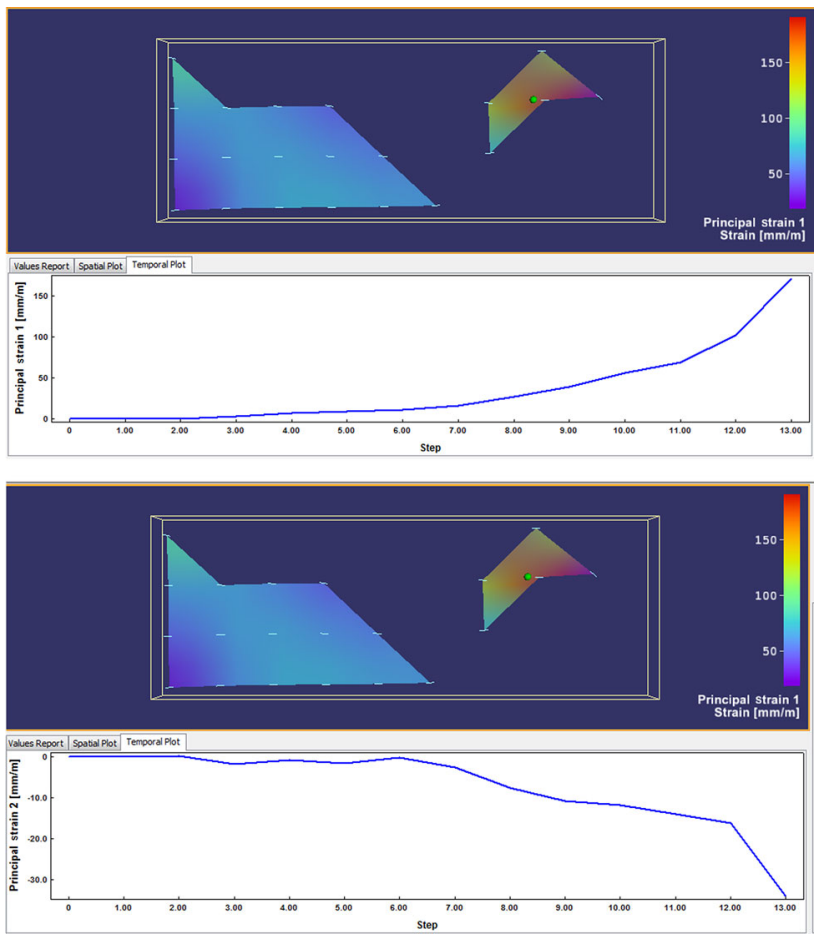

Fig. 16 History of main strain at dynamic fracture

\subsection{Scheme for verifying the constitutive parameters}

As shown in Fig. 17, the material parameters were verified by the penetration test of aluminum alloy profiles for high-speed trains. The test piece was a sandwich plate with a width of $100 \mathrm{~mm}$ and a height of $220 \mathrm{~mm}$, which is provided by CRRC Qingdao Sifang Co., Ltd. A slender bullet was fired through the Hopkinson rod's air gun. The diameter and length of the bullet are $16 \mathrm{~mm}, 300 \mathrm{~mm}$, respectively. The initial velocity of the bullet was measured by laser velocimetry. The remaining kinetic energy of the bullet is absorbed by the buffer device at the back end of the test piece.

The process of target penetration by bullet was recorded by high-speed photography. We obtained the morphology of the penetration process, which can be used to compare with the results of software calculation. We also obtained the residual velocity of the bullet by DIC analysis of the pictures taken, which can be used to verify the accuracy of energy absorption. We can verify the accuracy of constitutive parameters and dynamic failure strain by comparing the morphology and residual velocity.

The size of the model is the same as that in the physical experiment. Numerical calculation is carried out in LSDYNA explicit dynamic analysis software. The numerical calculation model is divided into three parts: bullets, aluminum alloy plates, and supports. The numerical model is shown in Fig. 18. 


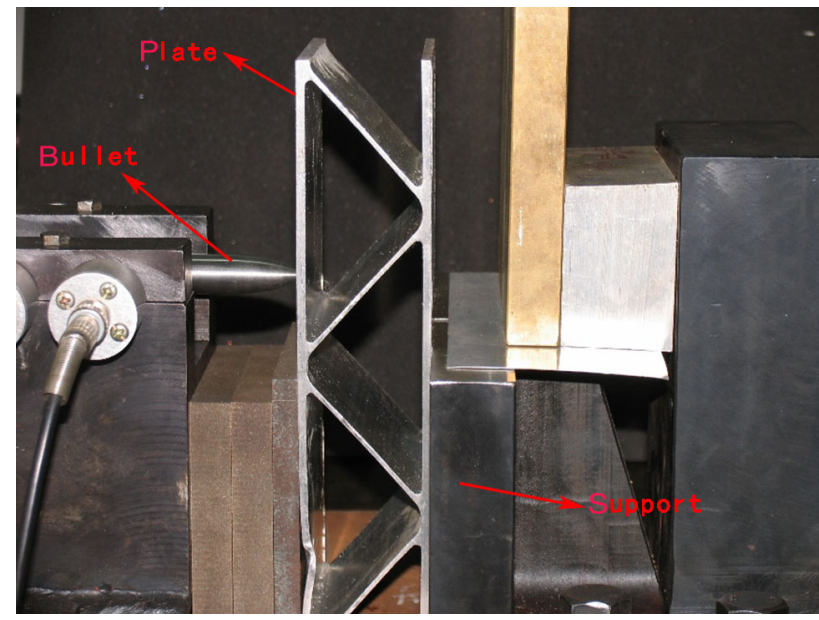

Fig. 17 Experimental verification platform

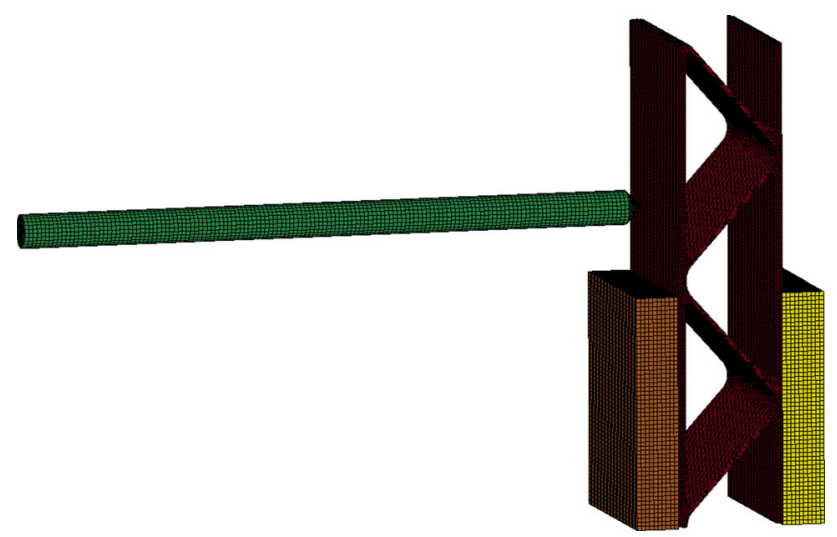

Fig. 18 Finite element model of the experimental verification platform

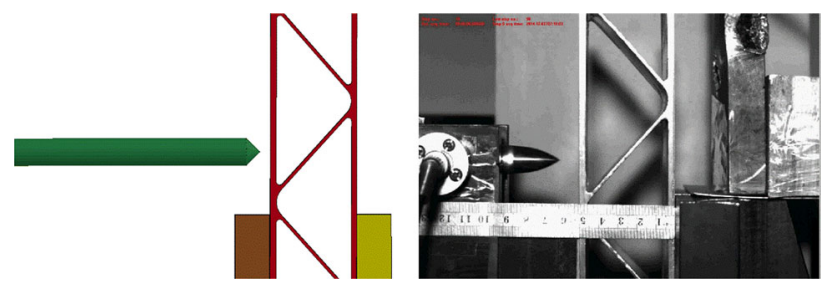

Fig. 19 Morphology of initial time

As shown in Fig. 19, the penetration position of the bullet is consistent with that in the experiment. The initial speed is set to $50 \mathrm{~m} / \mathrm{s}$, which is consistent with the speed measured by laser velocimetry in the experiment. The support is set to a rigid fixed block. The material parameters of each part are
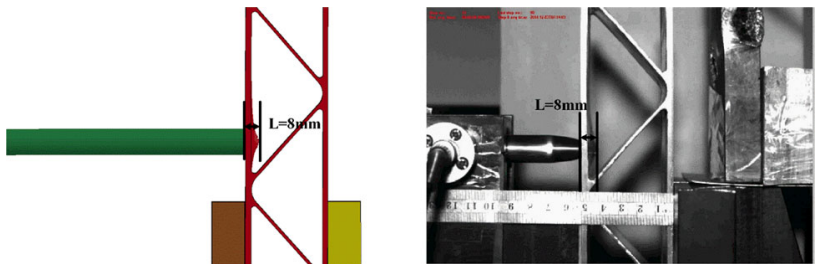

Fig. 20 Appearance of a bullet through the first layer

shown in Table 1. Because the bullets did not show obvious deformation in the real situation, and we don't care about the response of the bullet. The constitutive model of the bullet is chosen to be rigid. In order to reflect the actual boundary conditions, the friction coefficient between the support block and the aluminum alloy plate is set to 0.15 , which can make the lower end of the plate be fixed by the action of the supporting block.

\subsection{Comparison of damage morphology}

We have done the comparison of the damage morphology of the aluminum alloy in the experimental and calculated results. The accuracy of the constitutive parameters and the failure parameters is directly reflected by the morphology. Through qualitative and quantitative analysis, we compare the failure morphology obtained in different ways.

Before penetrated by bullets, the plate has a limit deformation in an undamaged area. The plate will be torn and damaged after continuing loading. This limit deformation can be described by the deflection. The maximum displacement between the inner surface and the outer surface is $L$. We set this distance as a measure of deflection. As shown in Fig. 20, the deflection calculated by the software is the same as the actual experiment. Their values are all $18 \mathrm{~mm}$. The consistency of deflection illustrates the accuracy of dynamic failure strain parameter.

As shown in Fig. 21, the first plate appears obvious tear deformation after penetrated by the bullet. The morphology of the surface tear is basically the same in the experiment and calculation results. Both showed petal-shaped destruction (there were three petals in the field of vision). Both open diameters are external diameter of bullet $(16 \mathrm{~mm})$. At this point, both values of $L$ are $18 \mathrm{~mm}$.

As shown in Fig. 22, the penetration of the bullet to the second plate is a typical oblique penetration. The most obvi-
Table 1 Material parameters of each part of the numerical mode

\begin{tabular}{llllllllll}
\hline Part & Model & $\rho\left(\mathrm{g} \cdot \mathrm{cm}^{-3}\right)$ & $E(\mathrm{GPa})$ & $v$ & $A(\mathrm{MPa})$ & $B(\mathrm{MPa})$ & $n$ & $C$ & $\varepsilon_{\text {eff }}$ \\
\hline Bullet & Rigid & 7.8 & 210 & 0.3 & - & - & - & - & - \\
Plate & J-C & 2.7 & 70 & 0.3 & 191.86 & 331.86 & 0.609 & 0.031 & 0.137 \\
Support & Rigid & 7.8 & 210 & 0.3 & - & - & - & - & - \\
\hline
\end{tabular}




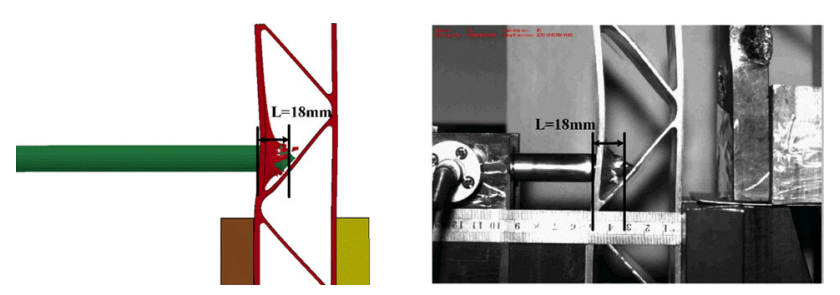

Fig. 21 Failure morphology of first layer plate surface
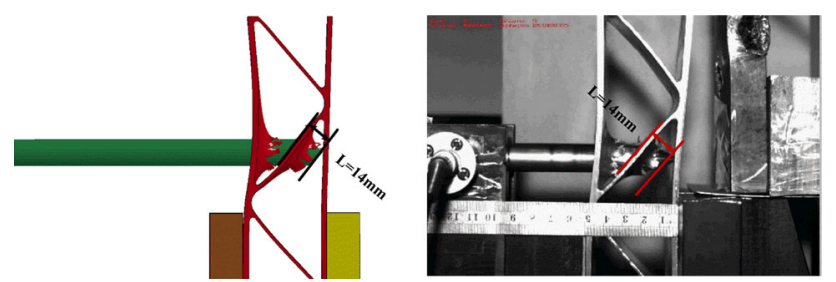

Fig. 22 Failure morphology of the second layer plate surface

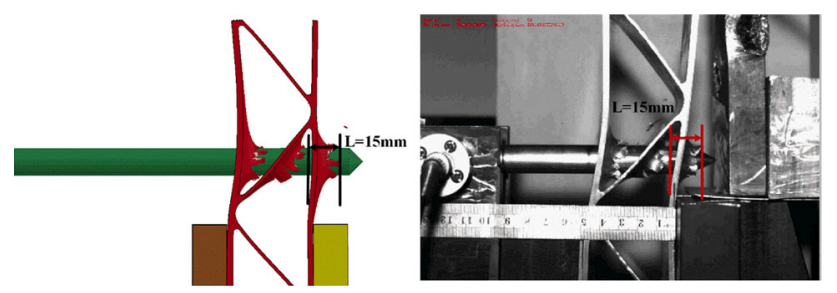

Fig. 23 Failure morphology of the third layer plate surface

ous feature is the asymmetry of material angle. The second plate also showed obvious tear deformation. The tear deformation of plate is petal destruction (one side is three petals in the view field, and the two gaps between the top two is larger). The material at the upper end is parallel to the bullet. The material at the lower end is parallel to the second plate after opening one angle. At this point, both values of $L$ are $14 \mathrm{~mm}$.

After the third plate was penetrated by bullets, the $L$ value of both was $15 \mathrm{~mm}$. As shown in Fig. 23, the morphologies of the petals are basically the same.

\subsection{Comparing the velocity history of bullets}

In the experiment, the initial velocity of a bullet passing through the target was obtained by laser velocimetry. In the numerical model, we also set the initial velocity of the bullet to $50 \mathrm{~m} / \mathrm{s}$, which ensure the consistency between the two. In the case of the same initial velocity, the remaining velocity of the bullet is the focus of our attention.

Through the DIC analysis of high-speed photography, we obtained the speed curve before and after the penetration of third plate. The velocity curve obtained from the experiment is compared with that extracted from the numerical simulation results. In Fig. 24, we can see that the bullet speed history in the experiment is agreement with the numerical

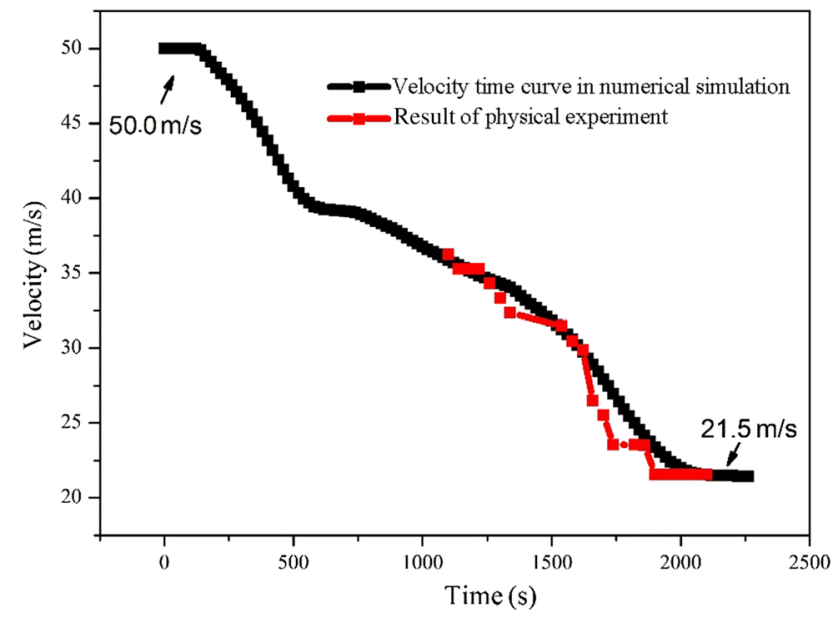

Fig. 24 Velocity-time curve of bullets

results. The final residual speed of the two is $21.5 \mathrm{~m} / \mathrm{s}$. The consistency of the speed history of the bullet also proves the accuracy of the constitutive parameters and the failure parameters.

\section{Conclusion}

The A6N01S-T5 aluminum alloy for a high-speed train body is studied in this paper. The static tension curve is obtained by the universal testing machine. The dynamic tensile curves of materials at different strain rates were obtained by using the integrated platform of Hopkinson rod automatic control and monitoring. Dynamic DIC technology is used to obtain the failure parameters of materials under dynamic impact. According to the static and dynamic test data, we can fit the constitutive model parameters that can be used in the numerical calculation of crash. The numerical calculation is carried out by LS-DYNA software. The simulation results are compared with the real impact penetration test. The accuracy of material constitutive parameters and dynamic failure strain parameters verified by comparing the failure morphology and the speed history of bullet. The main conclusions are as follows:

1. In the aspect of obtaining dynamic failure strain, we propose the method of SHTB + 3D DIC. Using a series of static and dynamic tests for the A6N01S-T5 aluminum alloy on high-speed trains, the parameters of JohnsonCook constitutive model and the dynamic failure strain parameters are obtained.

2. In the verification of the parameters, we use the sandwich structure of the real high-speed train body to carry out penetration test. The experimental results are compared with the simulation results: the accuracy of the parame- 
ters is verified by comparing the failure morphology of the structure and the speed history of bullet.

3. The experimental results provide a data basis for the crash simulation model of high-speed trains. The efficiency of structural design and optimization is improved.

Acknowledgements This work was supported by the National Department of Science and Technology (Grant 2016YFB1200505).

\section{References}

1. Chen, H.G., Administration, J.R.: Performance of the a6n01s-t5 aluminum alloy and the welding joint for high speed train at low temperature. Electr. Weld. Mach. 46, 77-82 (2016) (in Chinese)

2. Gou, G.Q., Huang, N., Chen, H., et al.: Research on corrosion behavior of A6N01S-T5 aluminum alloy welded joint for highspeed trains. J. Mech. Sci. Technol. 26, 1471-1476 (2012)

3. Yonetani, H.: Laser-mig hybrid welding to aluminium alloy carbody shell for railway vehicles. Weld. Int. 22, 701-704 (2008)

4. Yu, J., Gou, G., Zhang, L., et al.: Ultrasonic impact treatment to improve stress corrosion cracking resistance of welded joints of aluminum alloy. J. Mater. Eng. Perform. 25, 3046-3056 (2016)

5. Zhu, Z.Y., Chen, P., Zhou, H.M., et al.: Effect of the welding heat input on residual stresses in butt-weld of high-speed train. In: International Conference on Materials and Products Manufacturing Technology, Chengdu, October 28-30 (2011)

6. Dorn, J.E., Pietrokowsky, P., Tietz, T.E.: The effect of alloying elements on the plastic properties of aluminum alloys. JOM 2, 933-943 (1950)

7. Mrówka-Nowotnik, G.: Influence of chemical composition variation and heat treatment on microstructure and mechanical properties of 6xxx alloys. Arch. Metall. Mater. 46, 98-107 (2010)

8. Liu, Y.L., Kang, S.B., Kim, H.W.: The complex microstructures in an as-cast Al-Mg-Si alloy. Mater. Lett. 41, 267-272 (1999)

9. Irving, B.: Welding the four most popular aluminum alloys. Weld. J. 73(2), 51-55 (1994)

10. Bergsma, S.C.: Aluminum-magnesium-silicon alloy and treatment schedule, for use in the transport industry. US Patent 5961752-A (1999)

11. Troeger, L.P., Starke, E.A.: Microstructural and mechanical characterization of a superplastic 6xxx aluminum alloy. Mater. Sci. Eng. 277, 102-113 (2000)

12. Ito, T., Ishikawa, M., Otsuka, M., et al.: Ductility of 6xxx aluminum alloys at high temperature. J. Jpn. Inst. Ligh. Met. 53, 114-120 (2003)

13. Schulz, P., Berneder, J., Uffelmann, D., et al.: Advanced 5xxx-, $6 \mathrm{xxx}-$ and $7 \mathrm{xxx}$-aluminium alloys for applications in automotive and consumer electronics. Mater. Sci. Forum 690, 451-454 (2011)

14. Zhong, H., Rometsch, P.A., Cao, L.F., et al.: The influence of $\mathrm{Mg} / \mathrm{Si}$ ratio and $\mathrm{Cu}$ content on the stretch formability of $6 \times x \times$ aluminium alloys. Mater. Sci. Eng. 651, 688-697 (2016)

15. Koo, J.S., Cho, H.J.: Theoretical method for predicting the weight reduction rate of a box-type car body for rolling stock by material substitution design. Int. J. Automot. Technol. 10, 355-363 (2009)
16. Kang, S.G., Shin, K.B., Ko, T.H., et al.: Lightweight design of car bodies for double deck high-speed trains. J. Korean Soc. Manuf. Technol. Eng. 24, 177-185 (2015) (in Korean)

17. Gao, Y.H., Shi, X.F., Xie, S.M., et al.: Sensitivity analysis and lightweight design for high-speed train car body. J. Rail Way Sci. Eng. 14, 885-891 (2017) (in Chinese)

18. Rochard, B.P., Schmid, F.: Benefits of lower-mass trains for high speed rail operations. Proc. Inst. Civil Eng. Transp. 157, 51-64 (2004)

19. Wennberg, D., Stichel, S., Wennhage, P.: Benefits of weight reduction in high-speed train operations. ZEV Rail Glasers Annalen 137, 77-87 (2013)

20. Ezra, A.A., Fay, R.J.: An assessment of energy absorbing devices for prospective use in aircraft impact situations. in: Dynamic behaviour of structures, pp. 225-246. Pergamon, London (1972)

21. Yang, Z., Yu, Y.Y., Wei, Y.P., et al.: Crushing behavior of a thinwalled circular tube with internal gradient grooves fabricated by SLM 3D printing. Thin Walled Struct. 111, 1-8 (2017)

22. Li, Z.G., Yang, H.F., Hu, X.W., et al.: Experimental study on the crush behavior and energy-absorption ability of circular magnesium thin-walled tubes and the comparison with aluminum tubes. Eng. Struct. 164, 1-13 (2018)

23. Spigarelli, S., Evangelista, E., Mcqueen, H.J.: Study of hot workability of a heat treated AA6082 aluminum alloy. Scr. Mater. 49, 179-183 (2003)

24. Garrett, R.P., Lin, J., Dean, T.A.: An investigation of the effects of solution heat treatment on mechanical properties for aa $6 \mathrm{xxx}$ alloys: experimentation and modelling. Int. J. Plast 21, 1640-1657 (2005)

25. Werber, A., Liewald, M.: Influence of pre-strain and heat treatment on mechanical properties of aluminum sheet. Int. J. Mater. Form. 5, 307-315 (2012)

26. Zhong, H., Rometsch, P., Estrin, Y.: Effect of alloy composition and heat treatment on mechanical performance of 6xxx aluminum alloys. Trans. Nonferrous Met. Soc. China 24, 2174-2178 (2014)

27. Shi, L., Yang, H., Guo, L.G., et al.: Constitutive modeling of deformation in high temperature of a forging 6005A aluminum alloy. Mater. Des. 54, 576-581 (2014)

28. Zhu, D.J., Mobasher, B., Rajan, S.D., et al.: Characterization of dynamic tensile testing using aluminum alloy 6061-T6 at intermediate strain rates. J. Eng. Mech. 137, 669-679 (2011)

29. Vilamosa, V., Clausen, A.H., Børvik, T., et al.: Behaviour of $\mathrm{Al}-\mathrm{Mg}-\mathrm{Si}$ alloys at a wide range of temperatures and strain rates. Int. J. Impact Eng. 86, 223-239 (2015)

30. Zhang, D.N., Shangguan, Q.Q., Xie, C.J., et al.: A modified Johnson-Cook model of dynamic tensile behaviors for 7075-T6 aluminum alloy. J. Alloy. Compd. 619, 186-194 (2015)

31. Singh, R., Chauhan, S., Gope, P.C.: Fracture behavior of welded aluminum alloy at high strain rates. Proc. Eng. 173, 1246-1250 (2017)

32. Chen, X.Z., Peng, Y., Peng, S., et al.: Flow and fracture behavior of aluminum alloy 6082-T6 at different tensile strain rates and triaxialities. PLoS ONE 12, e0181983 (2017)

33. Sun, D.Z., Krawiec, M., Hooputra, H.: Characterization and modelling of the damage behavior of extruded aluminum profiles for crash simulations. Mater. Sci. Forum 877, 674-679 (2016)

34. Børvik, T., Hopperstad, O.S., Berstad, T.: On the influence of stress triaxiality and strain rate on the behaviour of a structural steel. Part II. Numerical study. Eur. J. Mech. A Solids 22, 15-32 (2003) 